\title{
Neurotransmitter Release from a Retinal Ribbon Synapse, a Modelling Study
}

\author{
Hassan Bassereh, Frank Rattay \\ Institute for Analysis and Scientific Computing, Vienna University of Technology, Wiedner Hauptstrasse \\ 8-10, 1040 Vienna, Austria \\ (e-mail: Hassanbassereh@gmail.com) \\ (e-mail:frank.rattay@tuwien.ac.at)
}

Keywords: differential equation, retinal bipolar cell, Monte Carlo calculation, random numbers, average values, spike, ribbon synapse

\section{INTRODUCTION}

The chemical synapse is a structure that permits information transfer from neuron to neuron via neurotransmitter release. Synapses of specialized cells in the retina and inner ear, e.g. retinal photoreceptor and bipolar cells or cochlear hair cells, have an extra electron-dense structure in comparison to ordinary synapses. This structure, called "ribbon", plays a vital role on modulating vesicle release in response to time dependent input signals. This input, in the following investigation the terminal membrane voltage $\mathrm{Vm}$ of a retinal bipolar cell, is related to the variation of light intensity at a specific small region in the retina. Neurotransmitter release in axon terminals starts when the terminal membrane is depolarized which leads to open the voltage-gated calcium channels. Opening of calcium channels increases intracellular calcium concentration which triggers transient vesicle release. Transient vesicle release refers to contrast adaption (Oesch and Diamond, 2011). In case of long pulse stimulation, transient release is replaced by a tonic or sustained release, which occurs at slower rate and refers to luminance adaption (Oesch and Diamond, 2011). On the other hand, role of different periodic pulses on transient neurotransmitter release is investigated. The pulses make the terminal membrane depolarized in different levels which leads to different amount of neurotransmitter release.

\section{RIBBON SYNAPSE}

We simulated a bipolar cell terminal that contains one ribbon and we computed the number of vesicles released from this ribbon for time dependent input. Vesicles combine to the ribbon as soon as any vesicle site is empty on the ribbon. Voltage-dependent calcium channels close to the ribbon support the release, Fig. 1. The open probability increases for these channels according to the increase of membrane voltage allowing calcium ions to come into the terminal. Intracellular calcium concentration $\left[\mathrm{Ca}^{2+}\right]$ is the key component in the

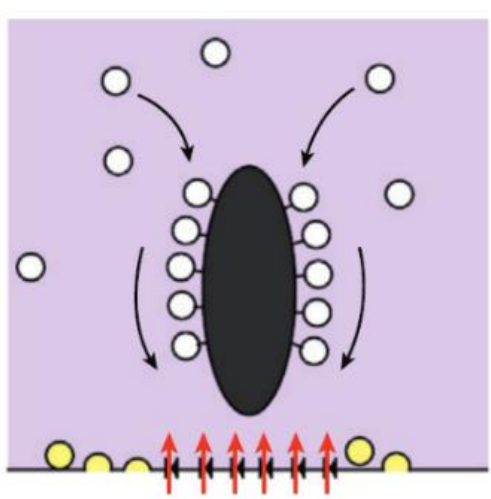

Fig, 1. Scheme of a ribbon synapse. The black oval-shaped part represents the ribbon and white spheres connected to the ribbon are 'ribbon vesicles'. Cytoplasmic vesicles are white spheres which are not connected to the ribbon. Red arrows show direction of incoming calcium ions. Picture taken from (Baden, et. al. 2013).

release process as calcium ions make the vesicles ready to be released by binding to them. When the transmembrane voltage increases, the internal calcium concentration increases (with some delay caused by the ion channel gating kinetics) which consequently leads to increment in the number of released vesicles. The vesicle release continues until the transmembrane voltage reaches about $-25 \mathrm{mV}$ (Oesch, and Diamond, 2011). On the other hand, the vesicle release has a stochastic component simulated as Monte-Carlo process.

\section{NEUROTRANSMITTER RELEASE}

The calcium concentration is governed by

$\frac{d\left[\mathrm{Ca}^{2+}\right]}{d t}=-\frac{i_{\text {ion }} A}{2 F V}-\frac{\left[\mathrm{Ca}^{2+}\right]-\left[\mathrm{Ca}^{2+}\right]_{\text {rest }}}{\tau}$

Where $\boldsymbol{i}_{\text {ion }}$ is the calcium current which represents L-type calcium channels, $\mathrm{A}$ and $\mathrm{V}$ are surface and volume of the terminal, respectively, $\mathrm{F}=96485.33 \mathrm{Cmol}^{-1}$ is the Faraday constant, $\left[\mathrm{Ca}^{2+}\right]_{\text {rest }}$ is the initial calcium concentration and $\boldsymbol{\tau}$ 
is the time constant of a passive extrusion process. The calcium current is explained by the Hodgkin-Huxley formalism (Hodgkin and Huxley, 1952). used in (Werginz, and Rattay, 2016) as follows: $\boldsymbol{i}_{C a}=\bar{g}_{C a} \boldsymbol{m}^{3}\left(V-E_{C a}\right)$ where $\overline{\boldsymbol{g}}_{\boldsymbol{C} a}$ is the maximum conductivity, $\mathrm{m}$ is the gating variable which represents open probability of the channel, $\mathrm{V}$ is the membrane voltage and $E_{C a}$ is the reversal potential and depends on the ratio of intracellular and extracellular calcium concentrations where the intracellular variations dominate.

A Monte-Carlo random process exerts the following condition on releasing: an equally distributed random integer number between 1 to 6 (number of initial vesicles) is selected and if there is any vesicle in that site, the neurotransmitter releasing occurs.

\section{DISCUSSION}

We fitted model parameter according to a synaptically twopaired pulse experiment in ref. (Oesch and Diamond, 2011, Graydon, et.al. 2014). The recorded data of a double pulse experiment demonstrates a nonlinear characteristic in vesicle release, Fig. 2, left. Our simulated results show averaged responses of 100 synapses, Fig. 2, right, which is fitted to the experimental data. In the next step, we simulated the vesicle release rate for periodic stimuli with frequencies of 2 and 8 $\mathrm{Hz}$, Fig 3. Note, some spontaneous vesicle release occurs, that is without any stimulation. Frequencies up to $20 \mathrm{~Hz}$ had the same characteristic features as the $8 \mathrm{~Hz}$ case (simulated but not shown). The model again calculated for an ensemble with 100 memberships and the pulses starts from $100 \mathrm{~ms}$ and continues to $1000 \mathrm{~ms}$.

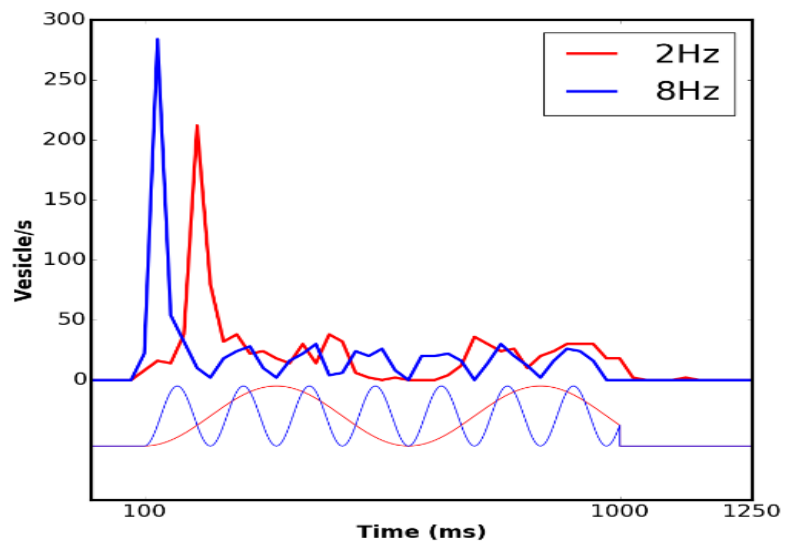

Fig. 3. Simulated vesicle release rate for two periodic input signals with frequencies of $2 \mathrm{~Hz}$ and $8 \mathrm{~Hz}$. The stimulus starts at $100 \mathrm{~ms}$ and continues to $1000 \mathrm{~ms}$. Some spontaneous vesicle release occurs, that is without any stimulation. Frequencies up to $20 \mathrm{~Hz}$ had the same characteristic features as the $8 \mathrm{~Hz}$ case (simulated but not shown).
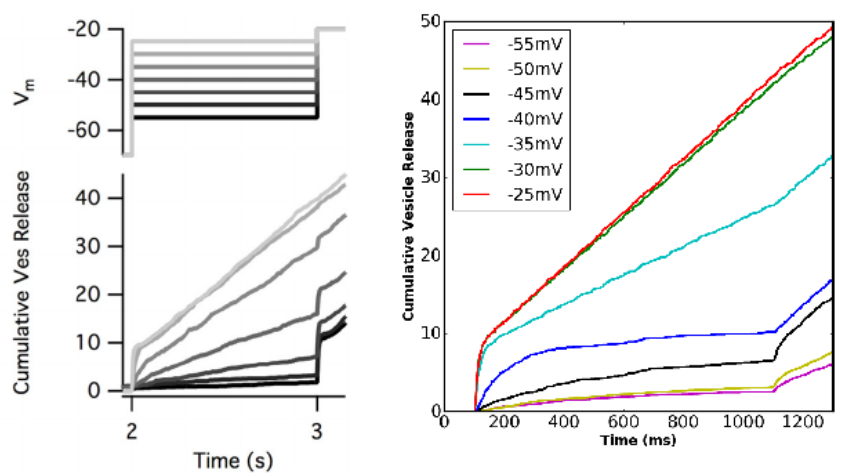

Fig. 2 (Left) Cumulative vesicle release (average of several experiments) vs. time in a single ribbon synapse (bottom) as response to a step function (top) Transmembrane voltage Vm was hold for one second at a constant value (e.g. $-55 \mathrm{mV}$ (darkest line) or $-25 \mathrm{mV}$ (bright line). A following higher voltage $(-20 \mathrm{mV})$ causes a sudden increase of release as long as the maximum release rate is not reached. (Graydon, et.al. 2014). (Right) The results from the model.

\section{CONCLUSIONS}

We used a calcium concentration dependent approach for neurotransmitter release for retinal bipolar cells. Direct use of membrane voltage as input to the ribbon model can be used instead of calcium concentration approach for slow events. However, there are different input output characteristics when graded potentials are interrupted by spikes in bipolar cells. When a spike reaches the terminal of a cell, calcium channels open and neurotransmitter will be released as consequence of an increase of intracellular calcium concentration. The presented model predicts the changing release rate as function of transmembrane voltage in the synaptic terminal both for spike trains and for graded potentials which can be assumed as arbitrary functions of time.

\section{REFERENCES}

Baden, T., Euler, T., Weckström, M., Lagnado, L. (2013) Spikes and ribbon synapses in early vision. Trends. Neurosci, 36, 480-488.

Graydon, CW. et. al. (2014) Passive diffusion as a mechanism underlying ribbon synapse vesicle release and resupply. J. Neurosci, 34, 8948-8962.

Hodgkin, A. L. and Huxley, A. F. (1952) A quantitative description of membrane current and its application to conduction and excitation in nerve. J. Physiol, 117, 500544.

Oesch, N.W., Diamond, J.S. (2011) Ribbon synapses compute temporal contrast and encode luminance in retinal rod bipolar cells. Nat. Neurosci, 14, 1555-1561.

Werginz, P. and Rattay, F. (2016) The impact of calcium current reversal on neurotransmitter release in the electrically stimulated retina. J. Neural. Eng, 13(4), 046013 . 